\title{
INNER SURFACE ROUGHNESS EVOLUTION AND SLIP DEFORMATION OF MICRO METAL TUBE DURING HOLLOW SINKING
}

\author{
${ }^{1,6}$ Saki SUEMATSU, ${ }^{1, *}$ Takuma KISHIMOTO, ${ }^{1}$ Hayate SAKAGUCHI, ${ }^{4}$ Kenichi TASHIMA, \\ ${ }^{5}$ Satoshi KAJINO, ${ }^{5}$ Shiori GONDO, ${ }^{1,2,3}$ Shinsuke SUZUKI \\ ${ }^{1}$ Department of Applied Mechanics and Aerospace Engineering, Faculty of Science and Engineering, \\ Waseda University, Shinjuku, Tokyo, Japan \\ ${ }^{2}$ Department of Materials Science, Faculty of Science and Engineering, Waseda University, Shinjuku, Tokyo, \\ Japan \\ ${ }^{3}$ Kagami Memorial Research Institute for Materials Science and Technology, Waseda University, Shinjuku, \\ Tokyo, Japan \\ ${ }^{4}$ Factory-Automation Electronics Inc., Osaka, Osaka, Japan \\ ${ }^{5}$ Advanced Manufacturing Research Institute, Department of Electronics and Manufacturing, National \\ Institute of Advanced Industrial Science and Technology (AIST), Tsukuba, Ibaraki, Japan \\ 6s suematsus@asagi.waseda.jp \\ "Present affiliation is Department of Mechanical and Biofunctional Systems, Institute of Industrial Science, \\ The University of Tokyo, Meguro, Tokyo, Japan
}

https://doi.org/10.37904/metal.2021.4100

\begin{abstract}
This study aimed to clarify the effect of the activity of the multiple slip systems on the height change of each grain of the inner surface of tube during the hollow sinking. A stainless steel tube with an outer diameter of $1.50 \mathrm{~mm}$, and a wall thickness of $0.045 \mathrm{~mm}$, which had only one grain across the wall thickness, was used as a starting material. The starting material was drawn without an inner tool in a single pass. The height unevenness and crystal orientation of the inner surface of the drawn tube were examined in the same measurement area using a laser microscope and an electron back scattered diffraction, respectively. The Schmid factor was calculated for each grain using the crystal orientation and the stress state which is the tensile and compressive in the drawing and transversal directions, respectively. The grains were generally convex when the calculated strain was positive, vice versa. The calculated strain is the sum of the deformations of the multiple slip systems with large Schmid factors where slip strain $\gamma$ was replaced with the Schmid factor. It was suggested that the height unevenness of the inner surface of the grains are caused by the multiple slip deformation in the drawing of micro metal tubes with a small number of grains across the wall thickness. Therefore, it is expected that the unevenness of the grains can be simply predicted.
\end{abstract}

Keywords: Hollow sinking, cold drawing, crystal deformation, EBSD, surface roughening

\section{INTRODUCTION}

Micro metal tubes are used for injection needles and heat exchangers [1], and are required to have a small diameter, thin wall, and smooth inner surface. In conventional hollow sinking, the increase in wall thickness was unavoidable. However, Kishimoto et al. proposed a method to control the drawing speed ratio at the entrance and exit side of the die, which enabled the hollow sinking of micro metal tubes without increasing the wall thickness [2]. Thus, hollow sinking process is suitable for the manufacturing of small-diameter tubes because it does not require an inner tool. However, the inner surface is simply roughened due to free surface 
roughening. Therefore, it is necessary to clarify the mechanism of surface roughening of the inner surface. Several studies [3-7] have been performed on the free surface roughening of sheet metals as follows.

Zhao et al. performed tensile tests and finite element method analysis of aluminum specimens with a single number of grains in the thickness direction. They reported that grains were deformed mainly due to the slip deformation in slip systems with maximum Schmid factor, and that the less constrained grains deformed significantly [3]. Balusu et al. performed uniaxial tensile tests on nickel polycrystalline sheets and crystal plasticity finite element method simulations in which grains were embedded in the homogeneous material and factors other than orientation were removed. As a result, it was found that the height change of each grain was approximately represented by several active slip systems, and that grains with loading directions close to $<001>$ and $<111>$ tended to sink [4]. Kubo et al. found that under equal biaxial tension, grains with a $\{001\}$ orientation sink due to their low deformation resistance and develop surface roughness due to the difference in deformation resistance between them and their surroundings [5]. Wouters et al. performed tensile tests on plates and found that the cumulative Schmid factor must be taken into account when multiple grains are included in the thickness direction [6]. On the other hand, the relationship between crystal orientation and inner surface roughness of tubes has been rarely studied due to the difficulty of measurement of the crystal orientation of the inner surface. However recently our research group has made it possible to measure the height distribution and crystal orientation of the inner surface of tubes by high-precision mirror polishing. Kishimoto et al. suggested that the $\{102\}$ plane was oriented in the thickness direction (ND) in the hollow sinking, where the wall thickness is controlled to decrease during drawing, thereby suppressing the development of surface roughness [7]. However, the relationship between the crystal orientation and the surface roughness evolution has not been clear.

Therefore, we conducted a new analysis of the from our previous experiments from the viewpoint of slip deformation. It is considered that the height unevenness of the inner surface can be expressed from the deformation of the crystal grains by multiple slip systems during the hollow sinking. This study aimed to clarify the effect of the activity of the multiple slip systems on the height change of each grain of the inner surface of the tube during the hollow sinking.

\section{EXPERIMENTAL METHOD}

The present experiment is the same as the one performed in our previous reserach [8]. A stainless steel tube with an outer diameter of $1.50 \mathrm{~mm}$ and a wall thickness of $0.045 \mathrm{~mm}$ was used as a starting material. The chemical composition of the tube is shown in Table 1. The starting material was manufactured by Fuji Seiko Co., Ltd. (Shizuoka, Japan) by drawing with plugs and annealed at $1273 \mathrm{~K}$ for $20 \mathrm{~s}$. The tube was additionally annealed at $1273 \mathrm{~K}$ for 60 min by Tamayakin Co., Ltd (Tokyo, Japan) to coarsen the crystal grains to have only one grain across the wall thickness.

Table 1 Chemical composition of the stainless steel (SUS304) tube used in this study (mass\%). This table is reprinted with permission from the publisher of the Ref. [6].

\begin{tabular}{|c|c|c|c|c|c|c|c|}
\hline $\mathbf{C}$ & $\mathbf{S i}$ & $\mathbf{M n}$ & $\mathbf{P}$ & $\mathbf{S}$ & $\mathbf{N i}$ & $\mathbf{C r}$ & $\mathbf{F e}$ \\
\hline 0.06 & 0.49 & 1.78 & 0.031 & 0.002 & 9.21 & 18.41 & Bal. \\
\hline
\end{tabular}

The starting material was drawn using a die with a diameter of $1.37 \mathrm{~mm}$ at a drawing speed ratio of 1.14 using a draw-bench machine (Factory-Automation Electronics Inc., Osaka, Japan) [6] shown in Figure 1.

The wall of the starting material and drawn tube were scraped to expose the inner surface. The microphotographs and height maps of the inner surface were obtained using a laser microscope (VK9510, Keyence, Miyagi, Japan). After that, the tubes were mirror-polished and crystal orientations were obtained using an electron back scattered diffraction (EBSD) (OIM7, TSL, Kanagawa, Japan) in the same field of view as the height map. 


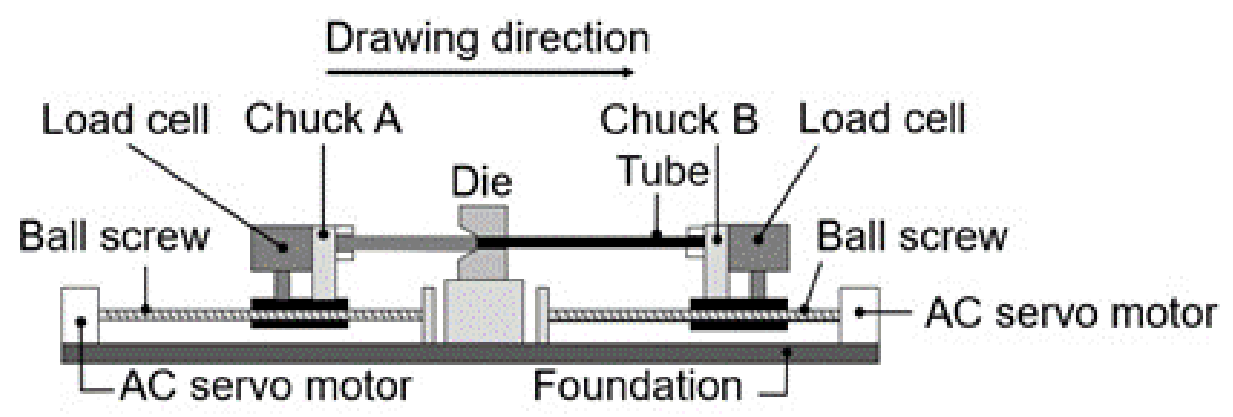

Figure 1 Schematic illustration of the draw-bench machine (Factory-Automation Electronics Inc.). This figure is reprinted with permission from the publisher of the Ref. [7].

\section{RESULTS}

Figure 2 shows the microphotographs, height maps, and IPF maps of the starting material and the drawn tube [7]. In the height map, the average height in the measurement field of view is defined as $0 \mu \mathrm{m}$, and the difference from that is defined as $h$. The IPF maps show the average orientation of each grain using MTEX [8], a toolbox of MATLAB ${ }^{\mathrm{TM}}$ (R2020a, MathWorks, Massachusetts, USA). The absolute value of the unevenness height in the height map increases, indicating that the inner surface was roughened during the hollow sinking. Lines that can be considered as grain boundaries were confirmed in the micrograph at almost the same positions as the grain boundaries shown on the IPF map. Thus, the unevenness of each grain can be determined using the height map. The grains are numbered to understand the correspondence between the height map and the IPF map simply.

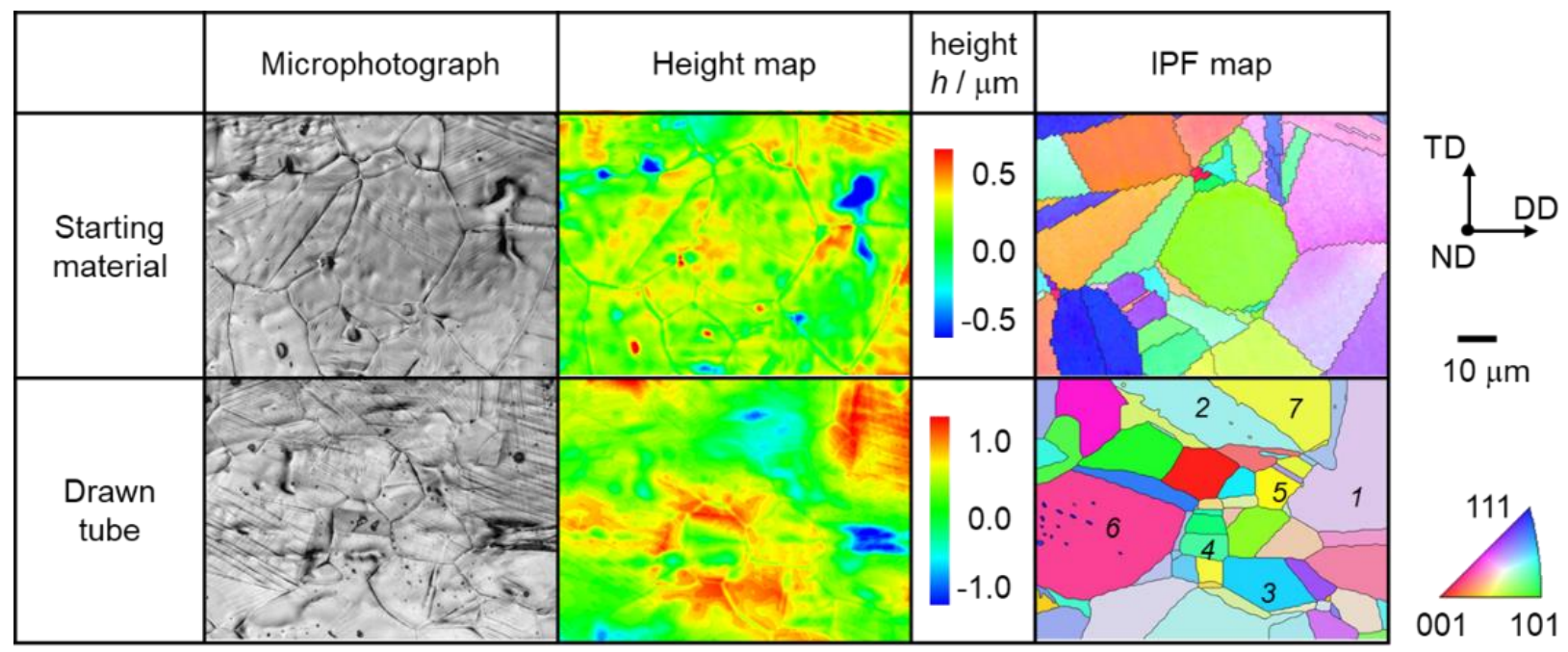

Figure 2 Microphotographs, height maps, and IPF maps of the inner surface of the starting material and drawn tubes in the same measurement area. The grains of interest are assigned a number (Grain-ID). The symbols DD, TD, and ND stand for the drawing direction, transversal direction, and normal direction to the inner surface, respectively. This figure is reprinted with permission from the publisher of the Ref. [7].

\section{DISCUSSION}

For the grains numbered in Figure 2, the relationship between the crystal orientation and the unevenness was examined. Figure 3 shows the schematic illustration of the relationship between the drawn tube and crystal grains. 
a)

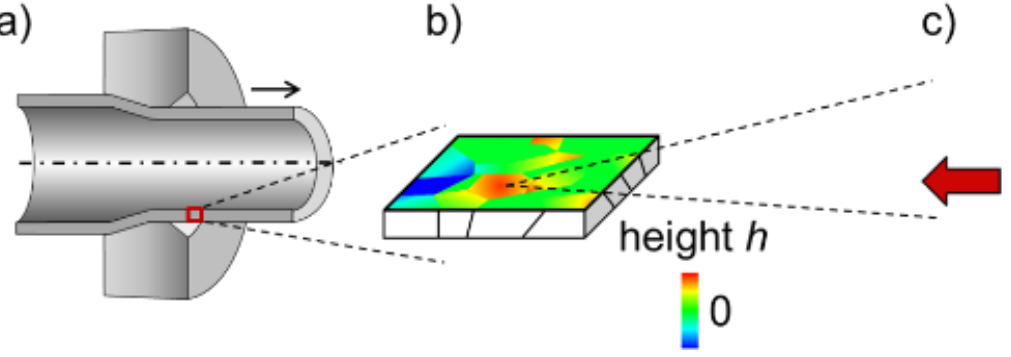

c)

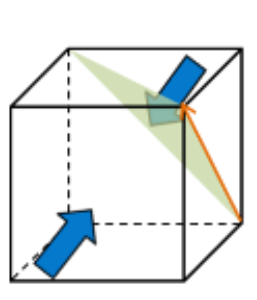

slip system

$(h k l)[u v w]$

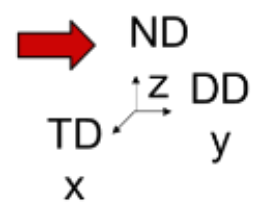

Figure 3 Schematic illustration of the relationship between the drawn tube and crystal grains: a) Schematic illustration of the hollow sinking, b) crystal grains of the drawn tube, and c) a slip system and stress state of a crystal. The red and blue arrows are tensile and compressive stress, respectively. The symbols DD, TD, and

ND stand for the drawing direction, transversal direction, and normal direction to the inner surface, respectively.

Table 2 shows the slip systems of FCC metal, one of which is SUS304. The Schmid factors $\left(S_{t}\right)$ for the slip systems for each grain were obtained using equation (1), where (hkl)[uvw] is a slip system $\alpha$ and [UVW] is the direction of principal stress. Here, the slip system is transformed to the sample coordinate system and normalized. For simplicity, it is assumed that the stress state on the tube was tensile in the drawing direction(DD), compressive in the transversal direction(TD), and negligible in the normal direction(ND) because it is sufficiently small compared to the stresses in DD and TD [7]. Therefore, [UVW] is defined as [-1 10$]$. As an example, the Schmid factors of Grain-1 are shown in Figure 4. Slip system No. is correspond to the No. in Table 2.

$S_{f}=\frac{(h U+k V+I M)(u U+v V+w W)}{\left(U^{2}+V^{2}+W^{2}\right) \sqrt{h^{2}+k^{2}+l^{2}} \sqrt{u^{2}+V^{2}+W^{2}}}$

Table 2 Slip systems of a FCC metal

\begin{tabular}{|c|c|}
\hline No. & Slip system \\
\hline 1 & $\left(\begin{array}{lll}1 & 1 & 1\end{array}\right)\left[\begin{array}{lll}0 & 1 & \overline{1}\end{array}\right]$ \\
\hline 2 & $\left(\begin{array}{lll}1 & 1 & 1\end{array}\right)\left[\begin{array}{lll}1 & 0 & 1\end{array}\right]$ \\
\hline 3 & $\left(\begin{array}{lll}1 & 1 & 1\end{array}\right)\left[\begin{array}{lll}1 & \overline{1} & 0\end{array}\right]$ \\
\hline 4 & $\left(\begin{array}{lll}1 & 1 & \overline{1}\end{array}\right)\left[\begin{array}{lll}1 & \overline{1} & 0\end{array}\right]$ \\
\hline 5 & $\left(\begin{array}{lll}1 & 1 & \overline{1}\end{array}\right)\left[\begin{array}{lll}1 & 0 & 1\end{array}\right]$ \\
\hline 6 & $\left(\begin{array}{lll}1 & 1 & \overline{1}\end{array}\right)\left[\begin{array}{lll}0 & 1 & 1\end{array}\right]$ \\
\hline 7 & $\left(\begin{array}{lll}\overline{1} & 1 & 1\end{array}\right)\left[\begin{array}{lll}0 & 1 & \overline{1}\end{array}\right]$ \\
\hline 8 & $\left(\begin{array}{lll}\overline{1} & 1 & 1\end{array}\right)\left[\begin{array}{lll}1 & 0 & 1\end{array}\right]$ \\
\hline 9 & $\left(\begin{array}{lll}\overline{1} & 1 & 1\end{array}\right)\left[\begin{array}{lll}1 & 1 & 0\end{array}\right]$ \\
\hline 10 & $\left(\begin{array}{lll}1 & \overline{1} & 1\end{array}\right)\left[\begin{array}{lll}1 & 0 & 1\end{array}\right]$ \\
\hline 11 & $\left(\begin{array}{lll}1 & \overline{1} & 1\end{array}\right)\left[\begin{array}{lll}1 & 1 & 0\end{array}\right]$ \\
\hline 12 & $\left(\begin{array}{lll}1 & \overline{1} & 1\end{array}\right)\left[\begin{array}{lll}0 & 1 & 1\end{array}\right]$ \\
\hline
\end{tabular}

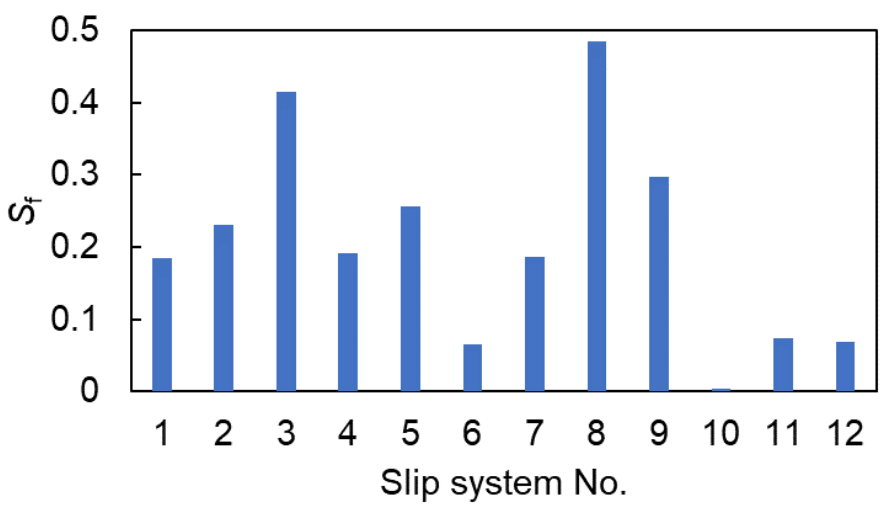

Figure 4 Schmid factors of Grain-1 in Figure 2. Slip system No. is correspond to the No. in Table 2.

Assuming that the strain is small and the lattice rotations are negligible, the grain deformation can be calculated using strain transformation [9]. Equation (2) shows the strain when a slip system $\alpha,(h k)[u v w]$ is active. The parameter $\gamma^{\alpha}$ is the slip strain. The strain in the inner surface direction is $w / \gamma^{\alpha}$. 


$$
\varepsilon^{\alpha}=\gamma^{\alpha}\left(\begin{array}{ccc}
u \cdot h & \frac{v \cdot h+u \cdot k}{2} & \frac{u \cdot l+w \cdot h}{2} \\
\frac{v \cdot h+u \cdot k}{2} & v \cdot k & \frac{w \cdot k+v \cdot l}{2} \\
\frac{u \cdot l+w \cdot h}{2} & \frac{w \cdot k+v \cdot l}{2} & w \cdot l
\end{array}\right)
$$

Manual calculation of $\gamma^{\alpha}$ considering time variation of stress and Euler angle is complicated and difficult. When the Schmid factor of a slip system is large, the slip strain $\gamma^{\alpha}$ tends to be large as well. Therefore, the slip strain $\gamma^{\alpha}$ was substituted for the Schmid factor in equation (2). Here, Figure 5 shows the schematic illustration of a crystal grain of the tube, ignoring the transversal direction. The lower surface is the die side and the upper surface is the inner surface. In this experiment, the wall thickness of the tube and the size of the crystal grain are the same. The red and blue lines represent the slip systems. When the grain is subjected to stress, slip deformation occurs in the slip system shown by the bold lines, and the grains deform as shown in Figure 5 a) to $b)$. The change in shape from a) to $b$ ) is judged to be concave, because the thickness of the grain after the deformation $t$ is smaller than the original thickness $t$. Here, we ignore the fine irregularities in the crystal grains and focus on the deformation of the grain as a whole, shown in gray. Although not represented in Figure 5 due to its simplicity, the grain can be convex when multiple slip systems are active in biaxial stress state in three dimensions.

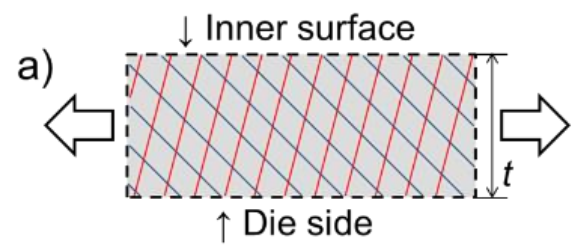

b)

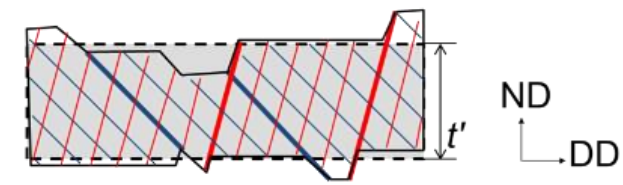

Figure 5 Schematic illustration of the crystal deformation caused by the activity of multiple slip systems represented by the red and blue lines in two dimensions. The gray areas surrounded by the dotted lines are the approximate shape of the entire crystal: a) Before the deformation. The arrows are tensile stress. $t$ is the original thickness of the grain, and b) after the deformation. Slip occurred on the slip systems of the bold lines. $t^{\prime}$ is the thickness of the grain after the deformation.

The same process is applied in three dimensions to consider the deformation toward the inner surface of the tube. The strain in the inner direction is calculated by adding up the strain of slip systems with large Schmid factors. The grain is convex when the value is positive, and concave when it is negative. Table $\mathbf{3}$ shows the calculated values and the unevenness obtained from the height map for each grain.

Table 3 Calculated strain in the inner surface direction and experimental results of unevenness of the crystal grains

\begin{tabular}{|c|c|c|c|}
\hline Grain-ID & Assumed strain: one slip system & Assumed strain: four slip systems & Unevenness \\
\hline 1 & 0.074 & 0.214 & Convex \\
\hline 2 & 0.060 & -0.025 & Concave \\
\hline 3 & 0.006 & 0.223 & Convex \\
\hline 4 & -0.031 & -0.180 & Flat (concave) \\
\hline 5 & -0.056 & 0.090 & Flat (concave) \\
\hline 6 & -0.007 & 0.030 & Convex \\
\hline 7 & 0.061 & -0.086 & Concave \\
\hline
\end{tabular}


Basically, we defined red-dominated grains as convex, blue-dominated grains as concave, and greendominated grains as flat in the height map shown in Figure 2. For flat grains, whether they are convex or concave compared to the surrounding grains is indicated in parentheses. A comparison of the assumed strains with the obtained unevenness of the heights shows that they agreed better when four slip systems were considered than when only one was considered. Therefore, it is suggested that the change in height of the inner surface of the grains could be approximately explained by adding up the deformations caused by several active slip systems calculated using the Schmid factor, where slip strain is replaced by Schmid factor.

\section{CONCLUSION}

When the strain calculated by adding up the deformations of multiple slip systems, where the slip strain $\gamma$ was replaced by the Schmid factor, is positive or negative, the grains are generally convex or concave, respectively. Therefore, it is expected that the unevenness of the grains can be simply predicted in the drawing of micro metal tubes with a small number of grains across the wall thickness.

\section{ACKNOWLEDGEMENTS}

The starting material used in this study was provided by Fuji Seiko Co., Ltd. This study was supported by the Kagami Memorial Research Institute for Materials Science and Technology of Waseda University. This study was supported by JXTG Nippon Oil \& Energy Corporation (grant number is B2R50Z004300). The Co-author Dr. Takuma Kishimoto performed this study as a Research Assistant under financial support from the Kagami Memorial Research Institute for Materials Science and Technology of Waseda University.

\section{REFERENCES}

[1] FAVIER, D., LIU, Y., ORGÉAS, L., SANDEL, A., DEBOVE, L., COMTE-GAZ, P. Influence of thermomechanical processing on the superelastic properties of a Ni-rich Nitinol shape memory alloy. Materials Science and Engineering A. 2006, vol. 429, no. 1-2, pp. 130-136.

[2] KISHIMOTO, T., GONDO, S., TAKEMOTO, K., TASHIMA, K., SUZUKI, S. Conditions for wall thickness reduction in hollow sinking of SUS304 tubes with drawing speed control in entrance and exit sides of die. Journal of Manufacturing Science and Engineering. 2019, vol. 141, no. 10, pp. 1-10.

[3] ZHAO, Z., RAMESH, M., RAABE, D., CUITIÑO, A. M., RADOVITZKY, R. Investigation of three-dimensional aspects of grain-scale plastic surface deformation of an aluminum oligocrystal. International Journal of Plasticity. 2008, vol. 24, no. 12, pp. 2278-2297.

[4] BALUSU, K., KELTON, R., MELETIS, E. I., HUANG, H. Investigating the relationship between grain orientation and surface height changes in nickel polycrystals under tensile plastic deformation. Mechanics of Materials. 2019, vol. 134, pp. 165-175.

[5] KUBO, M., NAKAZAWA,Y., HAMA, T., TAKUDA, H. Improvement of Surface Roughening Resistance of UltraLow-Carbon Steel Sheet by Reducing \{001\} Oriented Crystal Grains\{001\}. Tetsu-to-Hagané. 2018, vol. 104, no. 9, pp. 501-508.

[6] WOUTERS, O., VELLINGA, W. P., VAN TIJUM, R., DE HOSSON, J. T. M. Effects of crystal structure and grain orientation on the roughness of deformed polycrystalline metals. Acta Materialia. 2006, vol. 54, no. 10, pp. 28132821.

[7] KISHIMOTO, T., SUEMATSU, S., SAKAGUCHI, H., TASHIMA, K., KAJINO, S., GONDO, S. SUZUKI, S. Effect of crystal orientation on inner surface roughness of micro metal tubes in hollow sinking. Materials Science and Engineering A. 2021, vol. 805, no. January, p. 140792.

[8] BACHMANN, F., HIELSCHER, R., SCHAEBEN, H. Texture analysis with MTEX- Free and open source software toolbox. Solid State Phenomena. 2010, vol. 160, pp. 63-68.

[9] HOSFORD, W. F. Mechanical Behavior of Materials. Cambridge University Press. New YORK, 2010 , p. 419. 